\title{
Dependence of the open-closed field line boundary in Saturn's ionosphere on both the IMF and solar wind dynamic pressure: comparison with the UV auroral oval observed by the HST
}

\author{
E. S. Belenkaya ${ }^{1}$, S. W. H. Cowley ${ }^{2}$, S. V. Badman ${ }^{2}$, M. S. Blokhina ${ }^{1}$, and V.V. Kalegaev ${ }^{1}$ \\ ${ }^{1}$ Institute of Nuclear Physics, Moscow State University, Leninskie Gory, 119991 Moscow, Russia \\ ${ }^{2}$ Department of Physics \& Astronomy, University of Leicester, Leicester LE1 7RH, UK
}

Received: 11 September 2007 - Accepted: 17 December 2007 - Published: 4 February 2008

\begin{abstract}
We model the open magnetic field region in Saturn's southern polar ionosphere during two compression regions observed by the Cassini spacecraft upstream of Saturn in January 2004, and compare these with the auroral ovals observed simultaneously in ultraviolet images obtained by the Hubble Space Telescope. The modelling employs the paraboloid model of Saturn's magnetospheric magnetic field, whose parameters are varied according to the observed values of both the solar wind dynamic pressure and the interplanetary magnetic field (IMF) vector. It is shown that the open field area responds strongly to the IMF vector for both expanded and compressed magnetic models, corresponding to low and high dynamic pressure, respectively. It is also shown that the computed open field region agrees with the poleward boundary of the auroras as well as or better than those derived previously from a model in which only the variation of the IMF vector was taken into account. The results again support the hypothesis that the auroral oval at Saturn is associated with the open-closed field line boundary and hence with the solar wind interaction.
\end{abstract}

Keywords. Magnetospheric physics (Auroral phenomena; Magnetospheric configuration and dynamics; Planetary magnetospheres)

\section{Introduction}

Observations of the polar ultraviolet (UV) auroras at Saturn by the Hubble Space Telescope (HST) show that they generally form a ring around each pole, $\sim 1^{\circ}-3^{\circ}$ wide in latitude, located between $\sim 10^{\circ}-20^{\circ}$ co-latitude (Gérard et al., 1995, 2004; Cowley et al., 2004a; Badman et al., 2006). Cowley and Bunce (2003) and Cowley et al. (2004a) suggested, on the basis of theoretical discussion, that the magnetospheric

Correspondence to: E. S. Belenkaya

(elena@dec1.sinp.msu.ru) interaction with the solar wind leads to UV emission in the vicinity of the boundary between open and closed field lines, thus forming the auroral oval at Saturn. These results have subsequently been amplified by the modelling work of Cowley et al. (2004b), and also by the statistical analysis of the location the UV auroras presented by Badman et al. (2006). In January 2004 an opportunity arose to study the response of Saturn's auroras to variations in the interplanetary medium, when a sequence of UV images were obtained by the HST during a three-week interval, during the approach phase to Saturn of the Cassini spacecraft (Clarke et al., 2005; Crary et al., 2005; Badman et al., 2005; Bunce et al., 2006). The Cassini and HST data, reproduced here in Figs. 1 and 2 to be described further below, show that two corotating interaction region compressions, bounded by forward shocks, passed Saturn during the period of observations. After these compressions encountered Saturn's magnetosphere the auroral oval became brighter, especially at dawn, and contracted in radius. Belenkaya et al. (2007) have recently compared the images obtained in these compression regions with the location of the open-closed field line boundary calculated using the paraboloid model of Saturn's magnetospheric magnetic field (Alexeev et al., 2006; Belenkaya et al., 2006b), and have shown that good agreement is generally obtained. This study used a fixed magnetospheric magnetic field model based on the "Pioneer-11" model developed by Belenkaya et al. (2006b), with the behaviour of the open field region being studied by varying the direction and strength of the "penetrating" interplanetary magnetic field (IMF) vector based on observations made by Cassini. In the present paper we further develop this study by not only considering the influence of the prevailing IMF, but also the effect of the varying solar wind dynamic pressure observed by Cassini, which modulates the size of the magnetosphere and hence the other parameters that describe the model. 


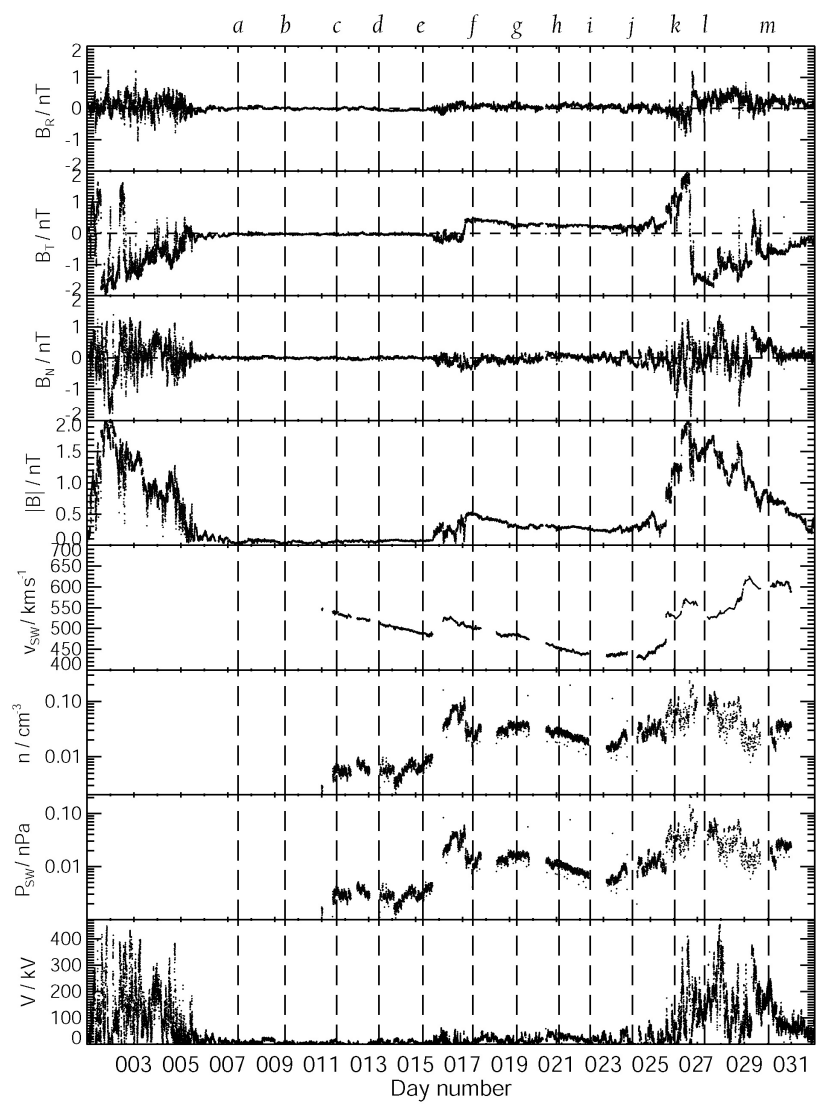

Fig. 1. Stacked plot of Cassini IMF and plasma data obtained during the January 2004 Cassini-HST campaign. The first four panels show the RTN magnetic field components $\left(B_{R}, B_{T}, B_{N}\right)$, and the magnetic field magnitude $|B|$ in nT. The fifth to seventh panels show the solar wind proton density $n_{p}\left(\mathrm{~cm}^{-3}\right)$, the solar wind velocity $v_{s w}\left(\mathrm{~km} \mathrm{~s}^{-1}\right)$, and the dynamic pressure $P_{S w}(\mathrm{nPa})$, respectively. The bottom panel shows the estimated magnetopause reconnection voltage $V(\mathrm{kV})$ using the algorithm of Jackman et al. (2004). The dashed vertical lines indicate the corresponding times of the HST images shown in Fig. 2, adjusted to take account of the solar wind propagation delay and the Saturn-HST light propagation delay. (Figure adapted from Badman et al. (2005).)

\section{Solar wind conditions}

Figure 1 shows the IMF and plasma data obtained by Cassini upstream of Saturn during the interval of HST observations in January 2004 (Crary et al., 2005; Badman et al., 2005). The top panels show the IMF components and magnitude in RTN coordinates, which is an orthogonal, right-handed spherical polar system referenced to the Sun's spin axis, with $B_{R}$ directed radially outward from the Sun, $B_{T}$ azimuthal about this axis in the direction of solar rotation and planetary motion, while $B_{N}$ completes the right-handed coordinate system (being directed positive northward in the equatorial plane). The magnetic models to be discussed below instead employ the IMF vector expressed in kronian solar-

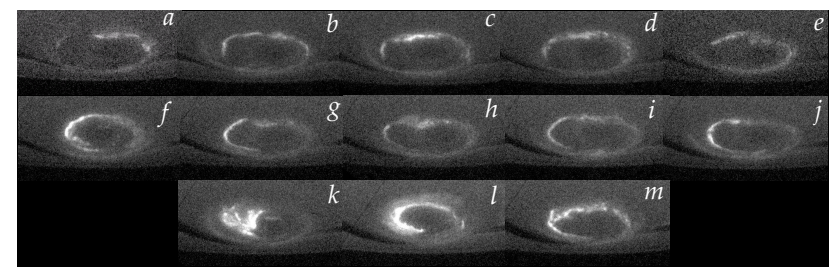

Fig. 2. UV images of Saturn's southern aurora obtained by HSTSTIS on $8,10,12,14,16,18,20,21,23,24,26,28$, and 30 January 2004 (panels $a$ to $m$, respectively). The panels have been generated by combining individual images obtained on a given HST orbit (Clarke et al., 2005; Bunce et al., 2006). The noon meridian is at the centre top of each plot, and dawn to the left. The dark arc seen in the lower portion of each image indicates the southern polar limb of the planet, with a portion of the rings seen behind. (Figure from Bunce et al., 2006).

magnetospheric (KSM) components $\left(B_{x}, B_{y}, B_{z}\right)$, where the $\mathrm{X}$-axis is directed toward the Sun, Saturn's magnetic moment lies in the $\mathrm{x}-\mathrm{z}$ plane, and $\mathrm{y}$ completes the right-handed orthogonal system. However, during the interval in question, the difference between the directions of the RTN and KSM axes is very small, with $B_{x} \approx-B_{R}, B_{y} \approx-B_{T}$, and $B_{z} \approx B_{N}$. Beneath this, in Fig. 1, are shown the velocity, density, and dynamic pressure of the solar wind, in which a "minor" compression region is seen to occur between 16-18 January, and a "major" compression region between 25 January and the end of the interval, separated by a rarefaction. The times corresponding to the HST images in Fig. 2 are indicated by the vertical dashed lines, where account has been taken of the $\sim 17$-h solar wind radial propagation delay from Cassini to Saturn, and the 68-min light travel time from Saturn to the HST. The solar wind delay is uncertain to within a few hours, however, due to possible non-radial propagation and the difference in helio-longitude of Cassini and Saturn (Crary et al., 2005), and this should be kept in mind in considering our results. During this interval Cassini was located near the ecliptic plane $\sim 0.2 \mathrm{AU}$ upstream of Saturn, and $\sim 0.5 \mathrm{AU}$ off the Sun-planet line toward dawn. The corresponding UV images in Fig. 2 show Saturn's southern auroral oval with the noon meridian at the centre top of each image, and dawn to the left (Clarke et al., 2005; Bunce et al., 2006). The scale of the oval relative to the planet can be readily discerned qualitatively from the dark arc seen in the lower portion of each image which indicates the southern polar limb of the planet, with a portion of the planet's rings seen behind. Quantitative information on the size and location of the UV oval will be given later in discussion related to Figs. 8 and 9.

As indicated above, the principal interplanetary parameters that influence the kronian magnetic model are the strength and direction of the IMF which penetrates the magnetosphere, and the solar wind dynamic pressure that modulates the size of the system through pressure balance. Belenkaya et al. (2006a, 2007) examined the interplanetary 
conditions observed near the (shifted) times of the compression region images $f, k$, and $m$ in Fig. 2, and concluded that the IMF vector can be characterised by the following KSM $\left(B_{x}, B_{y}, B_{z}\right)$ components, (i) $(0.0,-0.4,-0.4) \mathrm{nT}$ for image $f$ obtained shortly after the end of the "minor" compression region, (ii) $(0.5,-2.0,-1.4) \mathrm{nT}$ for image $k$ obtained just prior to the pressure maximum during the "major" compression, and (iii) $(-0.3,0.7,0.7) \mathrm{nT}$ for image $m$ obtained during the declining phase of the "major" compression. For simplicity in subsequent modelling, Belenkaya et al. (2007) used these IMF values together with a fixed magnetic field model considered to be representative of compression region conditions. The model employed was that derived earlier by Belenkaya et al. (2006a) based on conditions observed during the Pioneer-11 flyby, when the sub-solar magnetopause was compressed inward to $\sim 17.5 R_{S}$. (Here $R_{S}$ is Saturn's radius taken equal to $60330 \mathrm{~km}$.) According to the recent study of Cassini magnetopause positions by Arridge et al. (2006), the relationship between solar wind dynamic pressure $p_{s w}$ and the sub-solar radius of the magnetopause $R_{S S}$ is given empirically by

$p_{s w}(n P a) \approx\left(9.7 / R_{S S}\left(R_{S}\right)\right)^{4.3}$.

Thus a sub-solar radius of $\sim 17.5 \mathrm{R}_{S}$ corresponds to a dynamic pressure of $\sim 0.08 \mathrm{nPa}$. Comparison with Fig. 1 reveals that this value is indeed appropriate to image $k$ obtained during the early part of the "major" compression, so that reexamination of the results of Belenkaya et al. (2007) is unwarranted in this case. The measured dynamic pressures corresponding to images $f$ and $m$ are both lower than this, however, equal to $\sim 0.01$ and $\sim 0.03 \mathrm{nPa}$, respectively, such that the magnetosphere in these cases will have been somewhat expanded compared with the Pioneer-11 model employed by Belenkaya et al. (2007). In fact, Eq. (1) indicates that a dynamic pressure of $0.01 \mathrm{nPa}$ corresponds to a sub-solar magnetopause radius of $\sim 28 R_{S}$, while $0.03 \mathrm{nPa}$ corresponds to a radius of $\sim 22 R_{S}$. In this paper we thus model the open field region in these two cases, now taking into account not only the observed IMF vector, but also the observed solar wind dynamic pressure which modulates the size of the magnetosphere. In the following sections we thus find the set of model parameters corresponding to these conditions, and investigate the differences that arise compared with the results of the previous study.

\section{Selection of the input model parameters}

The kronian magnetospheric magnetic model employed here is that described by Alexeev et al. (2006) and Belenkaya et al. (2006b), representing a development of models derived previously for Earth and Jupiter (e.g. Alexeev et al., 2003; Belenkaya, 2004; Alexeev and Belenkaya, 2005). The mag-
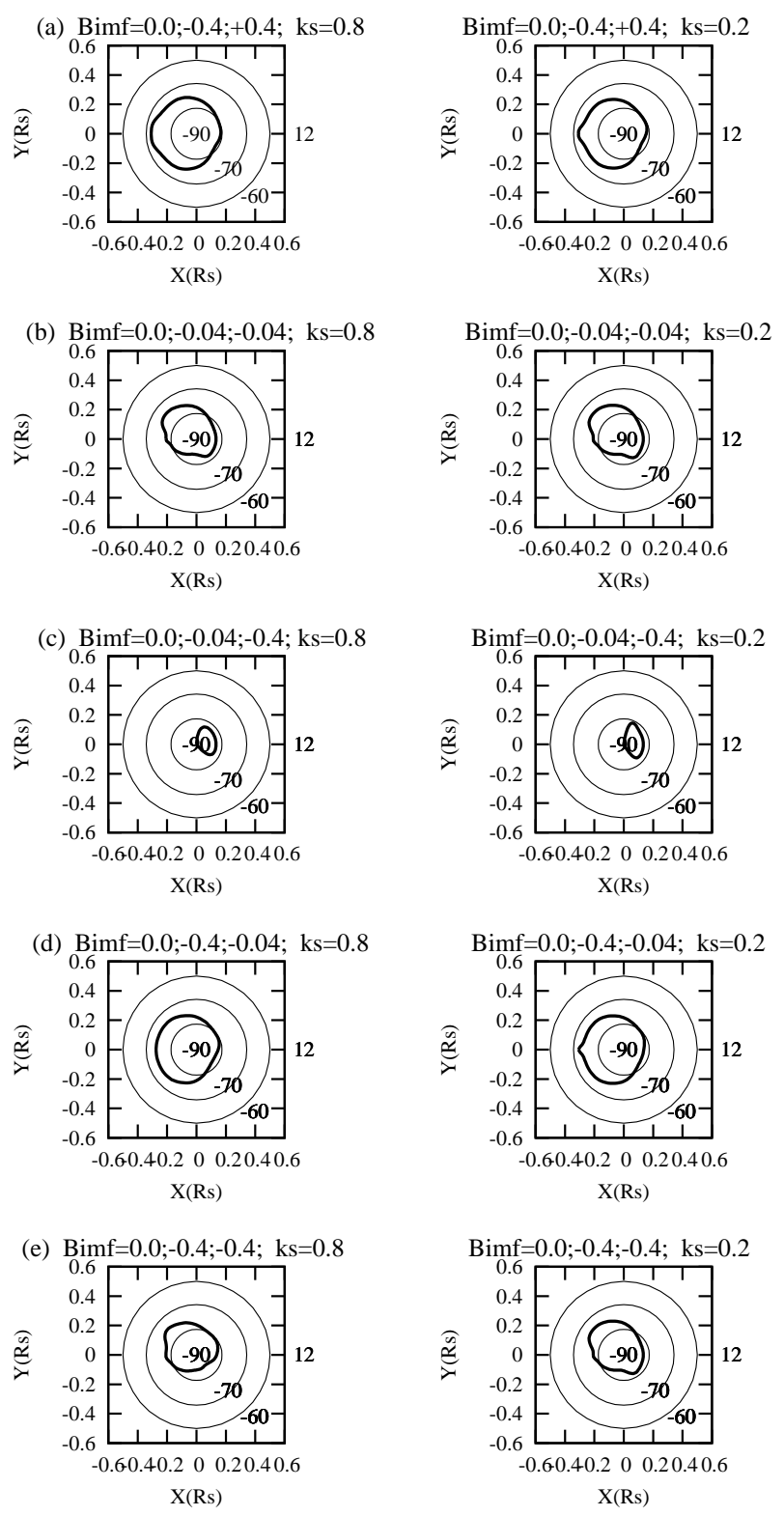

Fig. 3. Computed open field line regions in Saturn's southern ionosphere for the expanded magnetic field model appropriate to image $f$ whose parameters are given in the first column of Table 1, and for various penetrating IMF vectors. Each row shows results for a different IMF vector as indicated, for $k_{S}=0.8$ on the left and $k_{S}=0.2$ on the right. The view in each panel is looking through the planet onto the southern ionosphere, with noon to the right and dawn at the bottom. The bottom row corresponds to the specific IMF vector appropriate to image $f$.

netopause is taken to be a paraboloid of revolution about the Saturn-Sun line, given in KSM coordinates by

$x / R_{s s}=1-\left(y^{2}+z^{2}\right) / 2 R_{s s}^{2}$, 


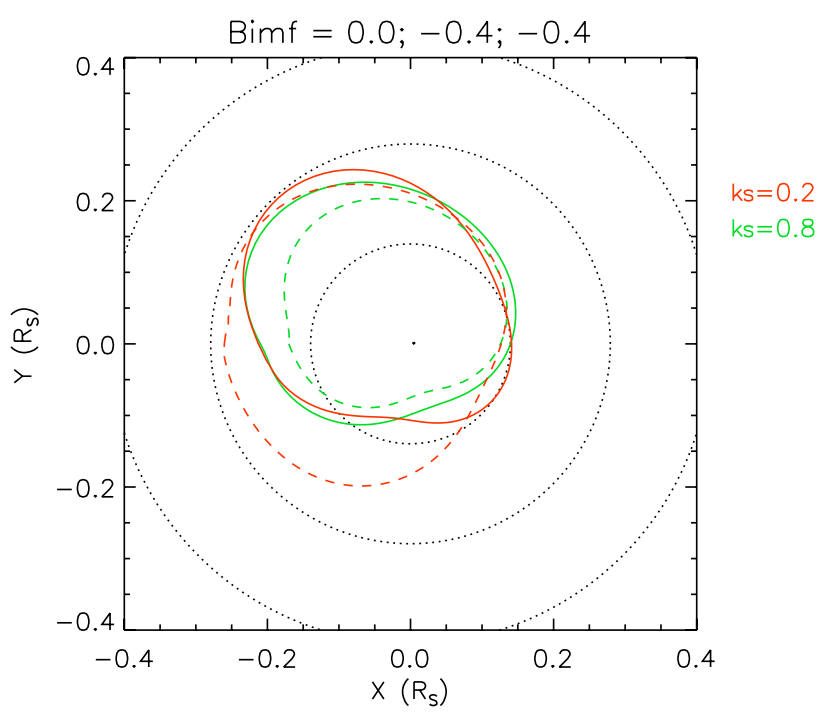

Fig. 4. Computed Southern Hemisphere open field line regions for the expanded magnetic model and IMF vector appropriate to image $f((0.0,-0.4,-0.4) \mathrm{nT})$ for $k_{S}=0.8$ (solid green line) and $k_{S}=0.2$ (solid red line), as shown in row (e) of Fig. 3. These are compared with related results obtained for the compressed magnetic model and the same IMF vector derived previously by Belenkaya et al. (2007), also shown for $k_{S}=0.8$ (dashed green line) and $k_{S}=0.2$ (dashed red line). The format is the same as for the panels of Fig. 3.

where $R_{S S}$ is again the sub-solar distance of the magnetopause. The contributors to the model magnetic field are (i) the intrinsic dipole field of the planet, together with the shielding current on the magnetopause which confines this field inside the magnetopause, (ii) the ring current, together with the shielding magnetopause current that similarly confines its field inside the magnetopause, (iii) the tail current and the corresponding magnetopause closure currents, and (iv) the IMF which partially penetrates into the magnetosphere. The ring current is modelled as a thin equatorial disc in which the azimuthal current intensity falls as the inverse square of the radial distance from the planet, while the tail current is re-scaled from an earlier terrestrial model.

The parameters which define Saturn's magnetospheric magnetic field in the model are thus the following: (i) $R_{s s}$ is the distance to the subsolar magnetopause, (ii) $\Psi$ is the tilt angle between the planet's magnetic dipole direction and the KSM $z$ axis $\left(~ 25^{\circ}\right.$ during the January 2004 interval, corresponding to Northern Hemisphere winter conditions), (iii) $R_{r c 1}$ and $R_{r c 2}$ are the distances to the outer and inner edges of the ring current, respectively, (iv) $B_{r c 1}$ is the radial component of the ring current magnetic field at the outer edge of the ring current, (v) $R_{2}$ is the distance from the planet to the inner edge of the magnetospheric tail current sheet, (vi) $B_{t} / \alpha_{0}$ is the field magnitude of the tail current at the inner edge of the tail current sheet, where $\alpha_{0}=\left(1+2 R_{2} / R_{S S}\right)^{1 / 2}$, and (vii) the effect of the IMF inside the magnetosphere is
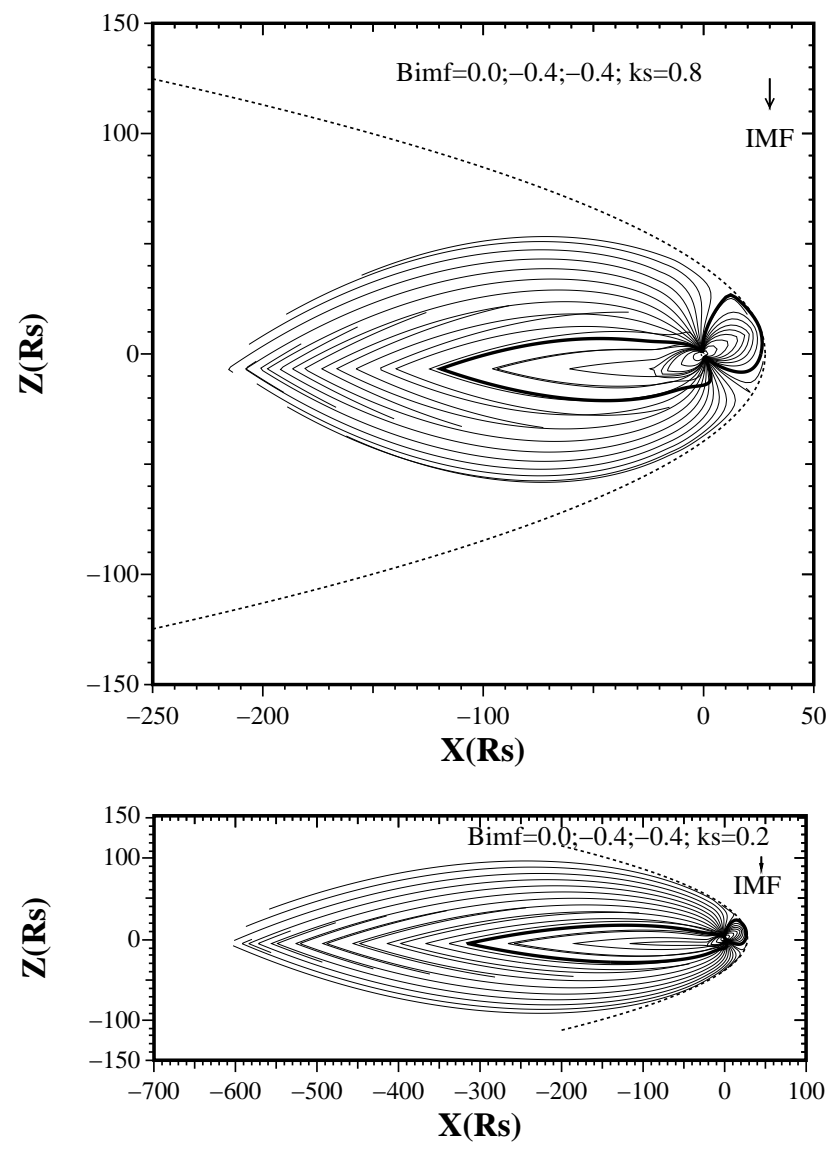

Fig. 5. Plots showing field lines emerging from Saturn's ionosphere on the noon-midnight meridian, projected into the noon-midnight $(\mathrm{x}, \mathrm{z})$ meridian plane, for the expanded magnetic model and IMF vector appropriate to image $f((0.0,-0.4,-0.4) \mathrm{nT})$, as indicated by the projected vector shown in the upper right-hand corner. The magnetopause is shown by the dashed curve, while the boundary between the open and closed field lines is shown by the bold curves. Panel (a) shows results for $k_{S}=0.8$, and panel (b) for $k_{S}=0.2$.

given by the uniform field $k_{s} \boldsymbol{B}_{\mathrm{IMF}}$, where $\boldsymbol{B}_{\mathrm{IMF}}$ is the IMF vector and $k_{S}$ is the coefficient of its penetration into the magnetosphere $\left(k_{S}<1\right)$. The actual value of $k_{S}$ is not accurately known at present. Here we therefore span the likely range by employing $k_{S}$ values of 0.2 and 0.8 in the calculations (see Belenkaya et al. (2007) and references therein).

The compressed Pioneer-11 model employed previously by Belenkaya et al. (2007) corresponds to the following set of parameters derived by Belenkaya et al. (2006b), $R_{S S}=17.5 R_{S}, R_{r c 1}=12.5 R_{S}, R_{r c 2}=6.5 R_{S}, B_{r c 1}=3.62 \mathrm{nT}$, $R_{2}=14 R_{S}$, and $B_{t}=8.7 \mathrm{nT}$, which we thus consider to be directly appropriate to image $k$. For image $f$, however, the observed dynamic pressure $\sim 0.01 \mathrm{nPa}$ implies a sub-solar radius of $\sim 28 R_{S}$, as noted above. For this value Alexeev et al. (2006) derived the following parameter set for the Cassini SOI orbit, $R_{s s}=28 R_{S}, R_{r c 1}=24.5 R_{S}, R_{r c 2}=6.5 R_{S}$, $B_{r c 1}=2.2 \mathrm{nT}, R_{2}=22.5 R_{S}$, and $B_{t}=5.3 \mathrm{nT}$, which we thus 
Table 1. Paraboloid model parameter sets appropriate to HST images $f, k$, and $m$.

\begin{tabular}{llll}
\hline $\begin{array}{l}\text { Model } \\
\text { parameters }\end{array}$ & $\begin{array}{l}\text { Expanded case (image } f) \\
p_{s w}=0.01 \mathrm{nPa}\end{array}$ & $\begin{array}{l}\text { Compressed case (image } k) \\
p_{s w}=0.08 \mathrm{nPa}\end{array}$ & $\begin{array}{l}\text { Intermediate case (image } m) \\
p_{s w}=0.03 \mathrm{nPa}\end{array}$ \\
& $\mathrm{IMF}=(0.0,-0.4,-0.4) \mathrm{nT}$ & $\mathrm{IMF}=(0.5,-2.0,-1.4) \mathrm{nT}$ & $\mathrm{IMF}=(-0.3,0.7,0.7) \mathrm{nT}$ \\
\hline$R_{s s}(\mathrm{Rs})$ & 28 & 17.5 & 22 \\
$\operatorname{Rrc} 1(\mathrm{Rs})$ & 24.5 & 12.5 & 15 \\
$\operatorname{Rrc} 2(\mathrm{Rs})$ & 6.5 & 6.5 & 6.5 \\
$B r c 1(\mathrm{nT})$ & 2.2 & 3.62 & 3 \\
$R_{2}(\mathrm{Rs})$ & 22.45 & 14 & 18 \\
$B t(\mathrm{nT})$ & 5.3 & 8.7 & 7 \\
\hline
\end{tabular}

also employ here with reference to image $f$. For image $m$, a dynamic pressure of $\sim 0.03 \mathrm{nPa}$ implies a sub-solar magnetopause radius of $22 R_{S}$, as noted above, for which we use the intermediate parameter set $R_{S S}=22 R_{S}, R_{r c 1}=15 R_{S}$, $R_{r c 2}=6.5 R_{S}, B_{r c 1}=3 \mathrm{nT}, R_{2}=18 R_{S}$, and $B_{t}=7 \mathrm{nT}$. The ring current parameters are similar to those derived by Connerney et al. (1983) using Voyager1 data, for which $R_{s s} \approx 23-24 R_{s}$, while the distance of the inner edge of the tail current is determined from the typical ratio $R_{2} / R_{s s} \approx 0.8$ (e.g. Alexeev et al., 2003, 2006; Alexeev and Belenkaya, 2005; Belenkaya et al., 2006b). These kronian parameter sets are summarized in Table 1 (the dipole tilt angle being $\Psi \approx 25^{\circ}$ in all cases). For simplicity of discussion below, these three models will be referred to as the expanded (image $f$ ), intermediate (image $m$ ), and compressed (image $k$ ) cases, corresponding to sub-solar magnetopause radii of 28,22 , and $17.5 R_{S}$, respectively, and to solar wind dynamic pressures of $0.01,0.03$, and $0.08 \mathrm{nPa}$.

\section{Model calculations}

The results derived previously by Belenkaya et al. (2007), using the compressed magnetic model, demonstrate that the open field region in Saturn's ionosphere depends strongly on the direction and strength of the penetrating IMF component. Specifically, the open flux increases with increasing positive IMF $B_{z}$ (northward), decreases with increasing negative IMF $B_{z}$ (southward), and shifts in the dawn-dusk direction in response to IMF $B_{y}$ (e.g. toward dusk in the Southern Hemisphere for $B_{y}$ negative). These effects are enhanced in the senses indicated as the penetration factor $k_{S}$ increases for a given IMF vector from small values towards unity. In Fig. 3 we demonstrate that similar effects are also found for the expanded model considered here, corresponding to the parameter set appropriate to image $f$. The figure shows plots of the open field region in Saturn's southern ionosphere (as for the UV images in Fig. 2), where the view is looking through the planet from the north, with noon to the right and dawn at the bottom. The circles indicate co-latitude from the southern pole in steps of $10^{\circ}$. Each row of the figure shows results for a given IMF vector, with the left column corresponding to $k_{S}=0.8$ and the right to $k_{S}=0.2$. Row (a) corresponds to a northward IMF, specifically $(0.0,-0.4,0.4) \mathrm{nT}$, showing an expanded open field region which is marginally larger for $k_{S}=0.8$ than for $k_{S}=0.2$, as expected. Rows (b) and (c) show how the open flux region becomes modified with increasing southward IMF in the case where IMF $B_{y}$ is small. Row (b) for $(0.0,-0.04,-0.04) \mathrm{nT}$ shows a contracted open field line region compared with row (a) which is similar for both values of $k_{S}$ (since the IMF vector is already small), while row (c) for $(0.0,-0.04,-0.4) \mathrm{nT}$ shows even further contraction, with the smallest open region occurring for $k_{S}=0.8$, as also expected. Rows (d) and (e) show the effect of the same variation in the $B_{z}$ component, but now with a larger negative $B_{y}$ of $-0.4 \mathrm{nT}$. Contraction of the open region again occurs, but not to the same extent as when $B_{y}$ is small, and the region shifts significantly toward dusk as $B_{z}$ becomes more negative.

The bottom row (e) in Fig. 3 employs both the specific dynamic pressure and IMF conditions that correspond to image $f$, i.e. the expanded field model and $(0.0,-0.4,-0.4) \mathrm{nT}$, respectively, such that we examine this case in more detail in Figs. 4 and 5. The solid lines in Fig. 4 compare the Southern Hemisphere open field regions for the two $k_{S}$ values in a plot having the same format as the panels of Fig. 3, but where the green line is for $k_{S}=0.8$ and the red line for $k_{S}=0.2$. It can be seen that the open regions are very similar to each other, but marginally more contracted for $k_{S}=0.8$ than for $k_{S}=0.2$. Figure 5 shows plots of the field lines in these two models that originate from the planetary ionosphere in the noon-midnight meridian, projected onto that plane, showing that the outer magnetosphere structure is strongly affected by the differing penetrating fields. In these plots the IMF vector is indicated in the top right-hand corner. The model magnetopause is shown by the dashed line, while the bold curves mark the boundary between open and closed field lines projected onto the $(\mathrm{x}, \mathrm{z})$ plane. The open field lines located south of this boundary are connected to the southern polar cap, and correspond to the area in Fig. 4 inside the solid green and red curves for $k_{S}=0.8$ and $k_{S}=0.2$, respectively. In Fig. 4 we also compare the open field regions with those derived previously by Belenkaya et al. (2007), using the same IMF vector and $k_{S}$ values, but with the compressed magnetic model. These are shown by the dashed lines, where again the green line is 


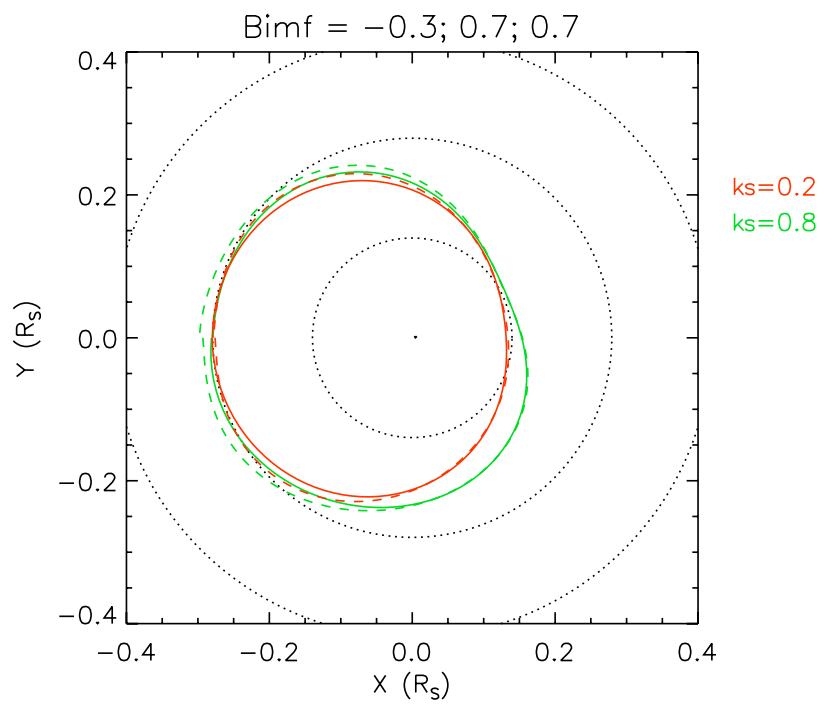

Fig. 6. As for Fig. 4, but now for the intermediate (solid lines) and compressed (dashed) magnetic field models and IMF vector $(-0.3$, $0.7,0.7) \mathrm{nT}$ appropriate to image $m$, for both $k_{S}=0.8$ (green) and $k_{S}=0.2$ (red).

for $k_{S}=0.8$ and the red for $k_{S}=0.2$. In this case the variation with $k_{S}$ is much larger than for the expanded model, such that the open region is more contracted than both of these when $k_{S}=0.8$, and more expanded when $k_{S}=0.2$.

In Figs. 6 and 7 we similarly consider results for the intermediate magnetic model together with IMF vector $(-0.3$, $0.7,0.7) \mathrm{nT}$, corresponding to image $m$. Figure 6 shows that the open region results are essentially similar for both intermediate and compressed field models, with a marginally larger open region occurring for $k_{S}=0.8$ than for $k_{S}=0.2$ in both cases, as expected for positive IMF $B_{z}$ conditions. The projected outer magnetosphere field lines shown in Fig. 7, in the same format as Fig. 5, again display a significant response to the change in the penetrating IMF field, though the form of the outer magnetosphere field differs greatly from the negative $B_{z}$ case.

\section{Comparison with observations}

We now directly compare the open field regions shown in Figs. 4 and 6 with the corresponding auroral distributions observed in images $f$ and $m$. In Fig. 8 we show results for image $f$, where the view is similar to previous ionospheric plots, except that the orientation has been rotated so that noon is at the bottom and dawn to the left. The dotted circles are again at intervals of $10^{\circ}$ co-latitude, and the auroral intensities are indicated by the colour scale shown on the right. Panel (a) corresponds to $k_{S}=0.8$ and panel (b) to $k_{S}=0.2$, where as in Fig. 4 the solid and dashed white lines in each case show results for the expanded and compressed field models, respectively, the latter thus corresponding to the re-
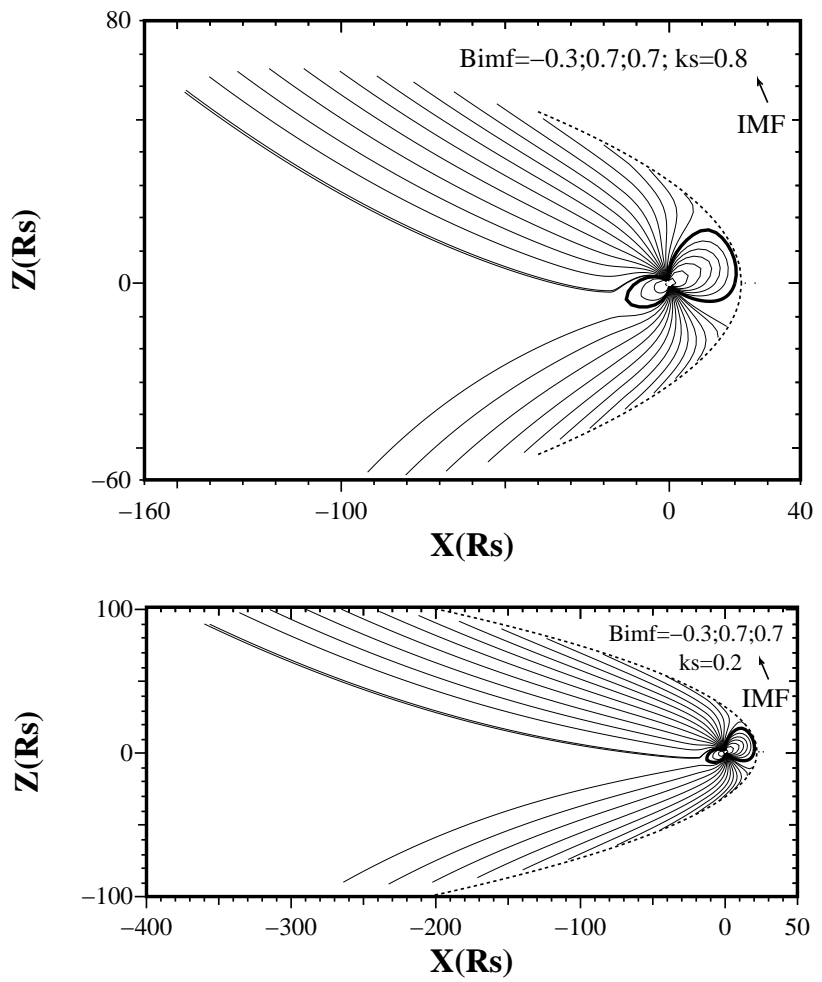

Fig. 7. As for Fig. 5, but now for the intermediate field model and IMF vector $((-0.3,0.7,0.7) \mathrm{nT})$ appropriate to image $m$. Panel (a) shows results for $k_{S}=0.8$, and panel (b) for $k_{S}=0.2$. As in Fig. 5, the magnetopause is shown by the dashed line, the boundary between open and closed field lines by the bold lines, and the projected IMF vector is indicated in the top right-hand corner.

sults presented previously by Belenkaya et al. (2007). It can be seen that the size and shape of the open field region derived here using the expanded field model agrees well with that of the dark region poleward of the auroras, and certainly for the case of $k_{S}=0.2$ agrees better than that derived previously using the compressed field model. In all cases, however, the model open regions are displaced somewhat to the nightside compared with the observed auroras. A tailward displacement of the open field region is expected under most interplanetary conditions, as shown, for example, in Fig. 3, due to the day-night asymmetry of the magnetosphere resulting from the flow of the solar wind. The origin of the sunward displacement of the auroras observed in image $f$ thus remains unclear at present.

In Fig. 9 we show results in the same format for image $m$, where now the solid and dashed white lines have been derived using the intermediate and compressed magnetic models, respectively, as in Fig. 6. In this case the differences between the results derived using the two magnetic models are much smaller, such that both are seen to agree well with the poleward border of the observed auroral distribution. 


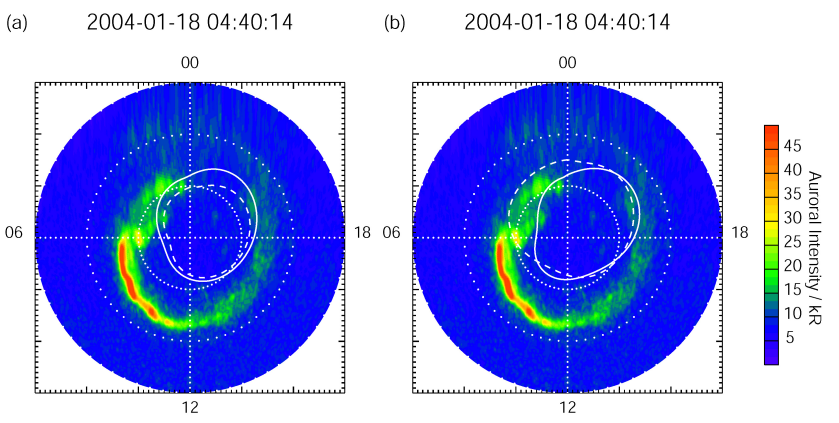

Fig. 8. HST UV image $f$ shown projected onto a polar grid from the pole to $30^{\circ}$ co-latitude, viewed looking through the planet onto the southern pole, as in Figs. 3 and 4, but with noon now at the bottom of the plot and dawn to the left as indicated. The UV auroral intensity is colour-coded according to the scale shown on the right-hand side of the figure. The over-plotted white lines show the modelled open field region, as in Fig. 4, where the solid lines show results obtained here using the expanded magnetic model and IMF vector $(0.0,-0.4,-0.4) \mathrm{nT}$, while the dashed lines show corresponding results obtained previously by Belenkaya et al. (2007) using the compressed magnetic model with the same IMF vector. Panel (a) shows results for $k_{S}=0.8$, and panel (b) for $k_{S}=0.2$.

\section{Conclusions}

In this paper we have extended the results presented previously by Belenkaya et al. (2007) on the size and shape of the open field line region in Saturn's ionosphere, and the related structure of the magnetosphere. In that study the open field region was modelled using the kronian version of the paraboloid magnetic model, and compared with images of Saturn's bright UV auroras obtained by the HST during solar wind compressions in January 2004. The modelling employed a penetrating IMF vector determined from simultaneous data obtained upstream of Saturn by the Cassini spacecraft, combined with an otherwise fixed magnetic field model representing compressed magnetospheric conditions. Here we have extended these results by also taking into account the observed dynamic pressure of the solar wind, which modulates the size of the magnetosphere and hence also other magnetospheric model parameters, such as the extent of the ring current and the tail current systems. Specifically, we have reexamined in detail two HST images for which the observed dynamic pressure was somewhat lower than implied by the compressed magnetospheric model employed by Belenkaya et al. (2007). The overall results obtained from this study are as follows.

(i) The kronian magnetosphere and open field line region in the ionosphere are sensitive to variations in the IMF strength and direction for both low and high solar wind dynamic pressure.

(ii) For southward-directed IMF, the variation in the modelled open field line region with IMF penetration coefficient $k_{S}$ is much smaller for low solar wind dynamic pressure than

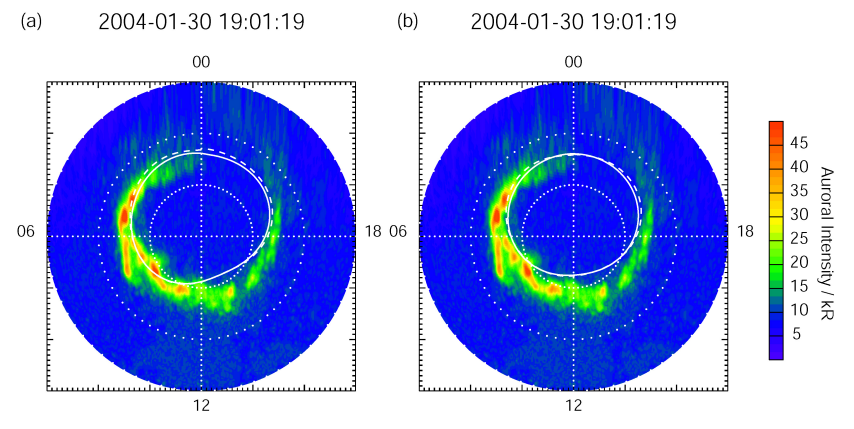

Fig. 9. Similar to Fig. 8, but now for HST image $m$. As in Fig. 6, the solid lines correspond to the intermediate magnetic model and IMF vector $(-0.3,0.7,0.7) \mathrm{nT}$, while the dashed lines correspond to the compressed magnetic model employed previously by Belenkaya et al. (2007) with the same IMF vector. Panel (a) shows results for $k_{S}=0.8$, and panel (b) for $k_{S}=0.2$.

for high dynamic pressure, the open region contracting with increasing dynamic pressure for high $k_{S}$, while expanding with increasing dynamic pressure for low $k_{S}$.

(iii) For northward-directed IMF, the open field region is relatively insensitive to variations in the IMF penetration coefficient $k_{S}$ and the solar wind dynamic pressure, though marginally expanding with increases in these parameters.

(iv) The agreement between the modelled open field region and the dark region poleward of the auroras observed by the HST remains as good as or better than that previously obtained using a fixed compressed magnetic model, thus again supporting the hypothesis that Saturn's auroral oval is related to the open-closed field line boundary, and hence to the solar wind interaction.

Acknowledgements. Work at the Institute of Nuclear Physics, Moscow State University, was supported by the RFBR Grants 0505-64435, 06-05-64508, and 07-05-00529. Work at Leicester was supported by STFC grant PP/E000983/1. S. W. H. Cowley was supported by a Royal Society Leverhulme Trust Senior Research Fellowship. The auroral data from which Figs. 2, 8, and 9 have been prepared are courtesy of J. Clarke (Boston University), and J.C. Gérard and D. Grodent (Université de Liège). The authors thank I. Alexeev for use of the Saturn magnetospheric model computer code employed here.

Topical Editor I. A. Daglis thanks S. M. Krimigis and another anonymous referee for their help in evaluating this paper.

\section{References}

Alexeev, I. I. and Belenkaya, E. S.: Modeling of the Jovian magnetosphere, Ann. Geophys., 23, 809-826, 2005, http://www.ann-geophys.net/23/809/2005/.

Alexeev, I. I., Belenkaya, E. S., Bobrovnikov, S. Y., and Kalegaev, V. V.: Modelling of the electromagnetic field in the interplanetary space and in the Earth's magnetosphere, Space Sci. Rev., 107, 7-26, 2003. 
Alexeev, I. I., Kalegaev, V. V., Belenkaya, E. S., Bobrovnikov, S. Y., Bunce, E. J., Cowley, S. W. H., and Nichols, J. D.: A global magnetic model of Saturn's magnetosphere, and a comparison with Cassini SOI data, Geophys. Res. Lett., 33, L08101, doi:10.1029/2006GL025896, 2006.

Arridge, C. S., Achilleos, N., Dougherty, M. K., Khurana, K. K., and Russell, C. T.:, Modeling the size and shape of Saturn's magnetopause with variable dynamic pressure, J. Geophys. Res., 111, A11227, doi:10.1029/2005JA011574, 2006.

Badman, S. V., Bunce, E. J., Clarke, J. T., Cowley, S. W. H., Gérard, J.C., Grodent, D., and Milan, S.E.: Open flux estimates in Saturn's magnetosphere during the January 2004 Cassini-HST campaign, and implications for reconnection rates, J. Geophys. Res., 110, A11216, doi: 10.1029/2005JA011240, 2005.

Badman, S. V., Cowley, S. W. H., Gérard, J.-C., and Grodent, D.: A statistical analysis of the location and width of Saturn's southern auroras, Ann. Geophys., 24, 3533-3545, 2006, http://www.ann-geophys.net/24/3533/2006/.

Belenkaya, E. S.: The Jovian magnetospheric magnetic and electric fields: Effects of the interplanetary magnetic field, Planet. Space Sci., 52, 499-511, 2004.

Belenkaya, E. S., Cowley, S. W. H., and Alexeev, I. I.: Saturn's aurora in the January 2004 events, Ann. Geophys., 24, 16491663, 2006a.

Belenkaya, E. S., Alexeev, I. I., Kalegaev, V. V., and Blokhina, M. S.: Definition of Saturn's magnetospheric model parameters for the Pioneer 11 flyby, Ann. Geophys., 24, 1145-1156, 2006b.

Belenkaya, E. S., Alexeev, I. I., Blokhina, M. S., Cowley, S. W. H., Badman, S. V., Kalegaev, V. V., and Grigoryan, M. S.: IMF dependence of the open-closed field line boundary in Saturn's ionosphere, and its relation to the UV auroral oval observed by the Hubble Space Telescope, Ann. Geophys., 25, 1-12, 2007, http://www.ann-geophys.net/25/1/2007/.

Bunce, E. J., Cowley, S. W. H., Jackman, C. M., Clarke, J. T., Crary, F. J., and Dougherty, M. K.: Cassini observations of the interplanetary medium upstream of Saturn and their relation to Hubble Space Telescope aurora data, Adv. Space Res., 38, 806814, 2006
Clarke, J. T., Gérard, J.G., Grodent, D., Wannawichian, S., Gustin, J., Connerney, J., Crary, F., Dougherty, M., Kurth, W., Cowley, S. W. H., Bunce, E. J., Hill, T., and Kim, J.: Morphological differences between Saturn's ultraviolet aurorae and those of Earth and Jupiter, Nature, 433, 717-719, 2005.

Connerney, J. E. P., Acuña, M. H., and Ness, N. F., Currents in Saturn's magnetosphere, J. Geophys. Res., 88, 8779-8789, 1983.

Cowley, S. W. H. and Bunce, E. J.: Corotation driven magnetosphere-ionosphere coupling currents in Saturn's magnetosphere and their relation to the auroras, Ann. Geophys., 21, 1691-1707, 2003 http://www.ann-geophys.net/21/1691/2003/.

Cowley, S. W. H., Bunce, E. J., and Prangé, R.: Saturn's polar ionospheric flows and their relation to the main auroral oval, Ann. Geophys., 22, 1379-1394, 2004a.

Cowley, S. W. H., Bunce, E. J., and O'Rourke, J. M.: A simple quantitative model of plasma flows and currents in Saturn's polar ionosphere, J. Geophys. Res., 109, A05212, doi: 10.1029/2003JA010375, 2004b.

Crary, F. J., Clark, J. T., Dougherty, M. K., Hanlon, P. G. Hansen, K. C., Steinberg, J. T., Barrachlough, B. L., Coates, A J., Gérard, J.-C., Grodent, D., Kurth, W. S., Mitchell, D. G., Rymer, A. M., and Young, D. T.: Solar wind dynamic pressure and electric field as the main factors controlling Saturn's auroras, Nature, 433, 720-722, 2005.

Gérard, J.-C., Dols, V., Grodent, D., Waite, J. H., Gladstone, G. R., and Prangé, R.: Simultaneous observations of the saturnian aurora and polar haze with the HST/FOC, Geophys. Res. Lett., 22, 2685-2688, 1995

Gérard, J.C., Grodent, D., Gustin, J., and Saglam, A.: Characteristics of Saturn's FUV aurora observed with the Space Telescope Imaging Spectrograph, J. Geophys. Res., 109, A09207, doi: 10.1029/2004JA010513, 2004

Jackman, C. M., Achilleos, N., Bunce, E. J., Cowley, S. W. H., Dougherty, M. K., Jones, G. H., and Milan, S. E.: Interplanetary magnetic field at $\sim 9 \mathrm{AU}$ during the declining phase of the solar cycle and its implications for Saturn's magnetospheric dynamics, J. Geophys. Res., 109, A11203, doi: 10.1029/2004JA010614, 2004. 